\title{
Incertidumbre socio-económica y actitudes hacia la inmigración en Europa
}

\author{
Antonio MARTÍN ARTILES \\ Universidad Autónoma de Barcelona, QUIT-IET \\ Antonio.Martin@uab.es \\ Óscar MOLINA \\ Universidad Autónoma de Barcelona, QUIT-IET \\ Oscar.Molina@uab.es \\ Guglielmo MEARDI* \\ Universidad de Warwick (Reino Unido) \\ Guglielmo.Meardi@wbs.ac.uk
}

Recibido: 27-01-2011

Aceptado: 09-03-2011

\begin{abstract}
RESUMEN
La actual crisis económica ha comportado un deterioro del mercado de trabajo en muchos países europeos, lo que ha estimulado la extensión de actitudes anti-inmigrantes entre la población. Estas actitudes antiinmigratorias se localizan fundamentalmente en los segmentos bajos del mercado de trabajo, aquellos de baja cualificación y de bajos salarios. En este artículo exploramos las actitudes de los ciudadanos europeos hacia los trabajadores inmigrantes. En particular tratamos de analizar que papel juega la incertidumbre socioeconómica en la conformación de las actitudes. Para ello comparamos los datos de las encuestas de los años 2002, 2008 y 2010 proporcionados por la Encuesta Social Europea para 17 países. Los resultados ponen de relieve la existencia de un cierto cambio de las actitudes entre ambos periodos. Las actitudes hacia la inmigración están condicionadas por tres tipos de variables: socio-económicas, ideológico-políticas y contextuales. El análisis de regresión logística pone de relieve el fuerte papel que juega la ideología izquierda versus derecha como predictor de las actitudes. Finalmente, el análisis de correspondencias múltiples nos ha permitido clasificar a los países estudiados según sus actitudes hacia la inmigración.
\end{abstract}

Palabras clave: crisis, incertidumbre económica, desempleo, inmigración, actitudes, políticas restrictivas, ideología política

* Proyecto de investigación Gusto SH-CT-2009-225301. Expresamos nuestro agradecimiento a Axel Van der Berg (McHill University, Montreal). Los dos primeros autores son miembros del Instituto de Estudios del Trabajo, Universidad Autónoma Barcelona, Meardi es profesor de la Universidad de Warwick. 


\title{
Socio-economic uncertainty and attitudes towards immigration in Europe
}

\begin{abstract}
The recent economic crisis has brought an unprecedented deterioration in labour market conditions in many European countries. This may have provided new stimulus for the extension of anti-immigrant attitudes amongst population, especially amongst those in the lower segments of labour markets. In this paper we analyze the attitudes of European citizens towards immigration and migrant workers. In particular, we try to understand what role plays perceived socio-economic uncertainty in order to explain attitudes towards immigration. Comparing data of the 2002, 2008 y 2010 waves of European Social Survey for sixteen European countries, the paper shows no remarkable change in the relative importance of objective and subjective socio-economic variables between the period of growth and recession. Moreover, logistic analysis consistently shows political-ideology to be a strong predictor of attitudes. Finally, multiple correspondence analyses allowed us to classify the countries surveyed according to their attitudes towards immigration.
\end{abstract}

Keywords: crisis, economic uncertainty, unemployment, immigration, attitudes, restrictive policies, political ideology

\section{REFERENCIA NORMALIZADA}

Artiles, M., Molina, O., Meardi, G. (2013). Incertidumbre socio-económica y actitudes hacia la inmigración en Europa. Cuadernos de Relaciones Laborales Vol. 31, núm. 1, p. 167-194.

SUMARIO: Introducción. 1. Análisis de las actitudes hacia la admisión de inmigrantes. 1.1. Incertidumbre. 1.2. Hipótesis. 2. Metodología. 2.1. Variables independientes. 2.2. Variable dependiente. 3. Descripción, tendencias y factores influyentes en las actitudes. 3.1. Incertidumbre socio-económica. 3.2. Influencia factores individuales. 3.3. Influencia factores socio-económicos. 3.4. Posicionamiento ideológico izquierdaderecha política, actitudes y valores. 3.5. Efecto país e influencia de las variables contextuales sobre las actitudes.4. Conclusiones y discusión. 4.1. Discusión. 5. Bibliografía.

\section{Introducción}

El objetivo de este estudio es analizar qué variables contribuyen a explicar las actitudes hacia la inmigración y, en particular, cuál es la importancia de la creciente incertidumbre ligada a la posición socio-económica de los individuos y derivada de la crisis económica actual. Partimos de la hipótesis que el deterioro de las condiciones de trabajo, el desempleo y los recortes en el Estado del Bienestar incrementan la percepción de incertidumbre socio-económica y la competencia por los escasos recursos de empleo y bienestar, lo que a su vez provoca una preferencia por políticas restrictivas en la admisión de trabajadores inmigrantes, así como actitudes hostiles, racistas y xenófobas (Mayda, 2006; Mau; Burkhardt, 2009, Balch, 2010). El interés de este estudio se justifica por la importancia que tiene la inmigración en Europa y por el crecimiento de las actitudes xenófobas en el contexto de crisis económica. La inmigración ha constituido un elemento central en la fase de expansión desde mediados de los años 90 hasta el 2007 y según la Comisión Europea lo seguirá siendo en el futuro inmediato como aportadora de movilidad entre los mercados de trabajo (European Commission, 2004, 2007; 2008; 2009; Zimmerman, 2009).

La actitud de la población hacia la admisión de inmigración es actualmente una cuestión de primer orden en la agenda político-social. En el corto plazo, porque determina el nivel de cohesión social y por tanto el conflicto entre diferentes grupos 
sociales. En el medio y largo plazo, porque influirá en el desarrollo de las políticas inmigratorias, tanto a nivel nacional como a nivel europeo. La actual crisis económica y sus consecuencias, como son el desempleo y la incertidumbre, ha estimulado la demanda de control restrictivo del flujo inmigratorio e incluso un cuestionamiento de la permanencia de trabajadores inmigrantes (véase Simon; Sikich, 2007; Balch, 2010; Cea D'Ancona; Valles, 2010). Ante la creciente importancia de éstos fenómenos, cabe preguntarse sobre los factores que inciden en las actitudes de la población hacia la inmigración y hasta qué punto la coyuntura de crisis y la mayor incertidumbre socio-económica han provocado un cambio significativo en las actitudes hostiles hacia la admisión de inmigrantes.

En este estudio hemos observado que las variables socioeconómicas relacionadas con la posición del individuo, como la edad, el nivel de estudios y los ingresos económicos, así como la competencia por el empleo y el bienestar tienen una notable capacidad explicativa lineal sobre las motivaciones de las actitudes restrictivas. Pero también las variables ideológicas ligadas al posicionamiento político izquierda-derecha y a las opiniones relacionadas con el compromiso igualitario tienen también una cierta capacidad predictiva (Schwartz, 2007). En efecto, quienes se sitúan a la izquierda tienen una actitud menos restrictiva que aquellos que se posicionan en la derecha política, lo que concuerda con el hallazgo de Saxton y Benson (2003) y con los análisis de Innerarity y Acha (2010) sobre el discurso nacionalista y anti-inmigratorio de la extrema derecha europea. Asimismo, las variables contextuales, como el índice de desigualdad social y la tasa de desempleo contribuyen de forma notable a explicar las actitudes restrictivas hacia la inmigración.

Este artículo se divide en cuatro partes. En la primera parte exploramos la literatura y el estado de la cuestión relacionado con las explicaciones que motivan las actitudes hacia la inmigración. En la segunda parte planteamos el desarrollo metodológico y explicamos las variables escogidas de la Encuesta Social Europea (ESE). En la tercera parte describimos los resaltados del análisis bivariados y regresiones. Y finalmente, en la cuarta parte apuntamos algunas conclusiones y planteamos cuestiones para la discusión sobre las políticas de inmigración.

\section{Análisis de las actitudes hacia la admisión de inmigrantes}

El estudio de las actitudes de la población hacia la admisión de inmigrantes ha ganado interés en los últimos años en el ámbito de la UE como consecuencia de la expansión hacia el Este y el aumento de la afluencia de trabajadores de países no miembros de la UE en los últimos años. En la literatura se puede encontrar un gran número de variables explicativas sobre las actitudes hacia la inmigración, que podemos agrupar en tres dimensiones: económicas, culturales-ideológicas y contextuales, aunque cabe decir que estas tres dimensiones interactúan entre sí (Balch 2010; Saxton, Benson 2003).

En primer lugar, encontramos en la literatura una explicación dominante en función de variables económicas como el desempleo, la pobreza y los salarios (Citrin, 
et al, 2005; Card et al. 2005; Ortega; Polavieja, 2009). La competencia de la inmigración por el empleo contribuye a que los autóctonos perciban un creciente deterioro en sus condiciones de trabajo, en la rebaja de salarios, aumento de la jornada laboral, la erosión de los derechos colectivos e incluso una pérdida en el poder de negociación de los sindicatos, como han observado González (2008) y Jódar, et. al. (2011) mediante análisis cualitativo con grupos de discusión. Desde la sociología también se ha estudiado el impacto de la inmigración y la reacción social en términos de competencias en relación a las "condiciones materiales objetivas" en la sociedad receptora (inserción en determinadas actividades laborales, acceso a la vivienda, acceso a los servicios sociales, etc.), lo que está asociado a tensiones y conflictos (Solé, et al. 2000). Estas explicaciones socio-económicas se centran en las implicaciones para el bienestar económico y laboral que tiene la inmigración, y en particular, la sensación de amenaza o vulnerabilidad que provoca para un creciente número de trabajadores en las sociedades postindustriales. De hecho, el reciente estudio de Cea D'Ancona y Valles (2010), pone de relieve como los brotes de xenofobia en España se explica por el incremento de la competencia por los menguados recursos de empleo y bienestar, lo que se está traduciendo en demandas de políticas restrictivas, tanto en la entrada como en la permanencia de inmigrantes.

En segundo lugar, hay otros estudios que han descubierto la importancia explicativa que tienen las variables relacionadas con los valores culturales y la ideología política en la conformación de las actitudes hacia la inmigración, como se ha observado desde la psicología social (Schwartz 1994; 2001). En efecto, los valores culturales tienen una importante capacidad explicativa en la aceptación o el rechazo de los inmigrantes, como han demostrado recientemente, entre otros, los estudios de Rodríguez Monter (2009) y Álvaro Estramiana y Rodríguez Monter (2010), quienes ponen de relieve que el perfil de los valores de las sociedades europeas sigue el siguiente orden, siguiendo la teoría de Schwartz (1994): Armonía, Compromiso Igualitario, Autonomía Intelectual; Conservación, Autonomía Afectiva y Competencia. Los países europeos conceden más importancia al Compromiso Igualitario que se expresa en valores normativos como la igualdad, la justicia social, el bienestar de los otros y la tolerancia (véase también Schwartz, 2007), lo que podría estar asociado hipotéticamente con actitudes algo más favorables, liberales, y abiertas hacia la entrada de inmigrantes. Asimismo, Rodríguez Monter (2009) señala que en los países europeos los tres criterios más importantes para explicar las actitudes hacia la inmigración son, por este orden, los aspectos culturales, la convivencia o relación directa y los laborales.

Por otra parte, desde la perspectiva de los valores ideológico-políticos, estudios como los de Saxton y Benson (2003) y Citrin et al. (2005) han puesto al descubierto el papel que juegan los valores ideológicos y la identificación política en las actitudes liberales o restrictivas hacia la inmigración, particularmente distinguiendo la orientación del voto en el espectro izquierda-derecha y las actitudes favorables y restrictivas hacia la inmigración. Estos aspectos han sido analizados por Innerarity y Acha (2010) en el ámbito europeo y muestran como los partidos de extrema derecha tienen un discurso focalizado en actitudes anti-inmigratorias y anti-universalista que 
les lleva a rechazar el multiculturalismo. Además, la inmigración es vista como "un problema", de ahí la creciente "politización de la inmigración", entrando a formar parte de los temas de la agenda política en los debates públicos, en los medios de comunicación y en el debate parlamentario (Balch, 2010).

A partir de recientes resultados electorales en diversos países europeos, se ha abierto un debate alrededor de la importancia de la ideología política versus la posición en el mercado de trabajo en lo referido a las actitudes hacia la inmigración. En algunos casos se ha apuntado la existencia de un cierto cambio en determinados segmentos del voto obrero ${ }^{1}$, en los territorios donde hay mayor concentración urbana de inmigrantes, hacia partidos de extrema derecha con posición antiinmigratoria debido a su percepción de competencia directa por los recursos de bienestar (vivienda, subsidios, pensiones, empleos, etc.) con el colectivo de trabajadores inmigrantes (Rink, et al. 2009). En pocas palabras, los inmigrantes aparecen relacionados de forma real o de forma imaginaria con todos los problemas sociales: el desempleo, la disolución cultural, el descenso del nivel educativo y de los servicios sanitarios, etc., (Innerarity y Acha (2009).

En tercer lugar, otros estudios han puesto énfasis en la influencia que tienen los elementos contextuales, como la tasa de desempleo, la tasa de inmigración, el PIB per cápita, el gasto en protección social y las instituciones del Estado de Bienestar. Estas variables contextuales ejercen influencias sobre las actitudes de la población acerca de la inmigración. Particularmente las políticas redistributivas de bienestar parecen haber contribuido a corregir las asimetrías y desigualdades que genera el mercado de trabajo y con ello han tendido a amortiguar las tensiones de la competencia por los recursos entre autóctonos e inmigrantes (Mayda, 2006; Morissen, 2008; Mau; Burkhardt 2009).

\subsection{Incertidumbre}

En el actual contexto, nos interesa especialmente analizar el efecto que las variables de tipo coyuntural económico pueden tener en la conformación de las actitudes. La crisis económica implica un aumento en la incertidumbre social y económica a que se ven expuestos los individuos. Sin embargo, éste aumento de la incertidumbre se distribuye de manera desigual, como señala Beck (1998:41). La forma en que la incertidumbre se distribuye en la sociedad sigue patrones de acuerdo con la posición socio-económica de los diferentes grupos sociales. Así pues, grupos que dentro de un determinado contexto social ocupan una posición relativamente fuerte o cómoda, pueden usar ésta posición para externalizar parte de la incertidumbre que un cambio en la coyuntura pueda causarles. Mientras que, por el contrario, los

\footnotetext{
${ }^{1}$ Por ejemplo, en la primera vuelta de las elecciones presidenciales francesas de 2012 la mayoría del voto obrero (32\%) fue dado al partido de extrema derecha de Le Pen, mientras que al Partido Socialista fue el $22 \%$ del voto obrero y el resto del voto se diluyó entre diferentes opciones políticas, según la encuesta IFOP publicada en El País, 23 abril 2012.
} 
grupos sociales con una débil posición socio-económica (como los desempleados, los jubilados y los trabajadores con contratos temporales) perciben el impacto de la incertidumbre y la competencia por recursos escasos.

De entre todos estos factores, el que ha adquirido más relevancia en el contexto actual es el referido a la percepción de incertidumbre y su distribución asimétrica entre trabajadores nativos e inmigrantes. Nótese aquí, que hablamos de incertidumbre y no de riesgo siendo el primer término más amplio e incluyendo la noción de riesgo. La idea de riesgo se refiere a un tipo de incertidumbre que es calculable y por tanto es posible protegerse a través de mecanismos diversos, incluyendo el recurso al mercado a través de instituciones financieras y aseguradoras. Sin embargo, hay otras muchas dimensiones de la incertidumbre que son incalculables y de difícil cobertura. En particular, el mercado de trabajo es fuente de incertidumbre, especialmente en un momento de fuerte crisis del empleo.

Las economías europeas se enfrentan hoy a una serie de presiones contradictorias en el marco de la incertidumbre de la economía global. Por un lado, se incrementa la demanda de flexibilidad en el mercado de trabajo al mismo tiempo, que por otro lado, la población demanda seguridad y las economías se vuelven más dependientes de un consumo estable (Crouch 2011). La crisis económica actual no ha hecho más que acentuar la tendencia de los problemas descritos: el aumento del desempleo, los problemas de redistribución de rentas y el incremento de la incertidumbre tienden a intensificar la competencia por los recursos de empleo y bienestar entre nativos e inmigrantes y por ende el conflicto social latente.

Históricamente las instituciones del Estado del Bienestar han contribuido a mitigar y corregir las desigualdades sociales y por tanto a incrementar la cohesión social y reducir el conflicto social. Sin embargo, los estados de bienestar se han asentado en una fuerte solidaridad nacional sustentados por poblaciones étnicamente homogéneas (Habermas, 2000). Al mismo tiempo, el incremento de la inmigración en las últimas décadas ha dado lugar a la aparición de nuevos problemas de integración social y de solidaridad como consecuencia de la diferenciación étnica y por origen de los ciudadanos (Mau; Burkhardt 2009). Algunos autores (Alesina; Glaeser, 2004; Sanderson, 2004; Soroka et al. 2006) asumen que la heterogeneidad étnica debilita al Estado de Bienestar en el ámbito de la Unión Europea. Incluso asumen la idea de que los estados reducirán eventualmente el gasto social como consecuencia de la presión de la diversidad étnica y del debilitamiento de los lazos de solidaridad.

\subsection{Hipótesis}

A partir de lo anterior, podemos establecer tres hipótesis que guían el análisis de la influencia de la incertidumbre socio-económica sobre las actitudes de admisión de inmigrantes.

En primer lugar (H1), la incertidumbre generada en un contexto de crisis económica, contribuye a acrecentar la percepción de competencia por el reparto del empleo y los beneficios de protección social entre nativos e inmigrantes. La percepción subjetiva de incertidumbre ligada a la posición socio-económica es mayor para determinados colectivos como son los trabajadores con contratos temporales, para 
los desempleados, así como aquellos que perciben bajos salarios y tienen un nivel de renta familiar bajo.

En segundo lugar (H2), el posicionamiento ideológico izquierda-derecha también contribuye a la conformación de las actitudes, de modo que quienes se sitúan a la izquierda tienen actitudes más favorables (liberales) y quienes se sitúan a la derecha tienen actitudes más restrictivas ante la entrada de inmigrantes. A ello añadimos como subhipótesis que aquellos que tienen valores políticos más igualitaristas tienen actitudes más liberales hacia la inmigración.

$\mathrm{Y}$, en tercer lugar (H3), el contexto de incertidumbre contribuye a la conformación de las actitudes: altas tasas de desempleo, de inmigración y de pobreza contribuyen a una demanda de políticas restrictivas, lo que explica la diferencia entre países europeos.

\section{Metodología}

En esta investigación hemos utilizado la Encuesta Social Europea (ESE) para analizar el efecto de la percepción subjetiva de incertidumbre socio-económica sobre la valoración de la inmigración. Para poder analizar el efecto que ha tenido la crisis económica hemos utilizado los datos correspondientes a los años 2002 (periodo de crecimiento), 2008 (inicio periodo de crisis) y 2010 (apogeo de la crisis). La comparación entre tres años en los cuales la situación económica ha cambiado de forma significativa, puede ayudarnos a entender el alcance real de variables contextuales y su influencia en la percepción de incertidumbre ligada a la inmigración. En la encuesta del año 2002 participaron 32.805 personas, en la de 2008 participaron 34.470 personas y en la encuesta del 2010 participaron 39.723 personas en los diecisiete países estudiados, siempre con un tamaño muestral superior a 1.500 personas por país participante en todos los años. La ESE no es una encuesta de panel, pero a pesar de ello nos puede facilitar una aproximación a las tendencias de las actitudes, como lo ha hecho atinadamente Feltzer (2011).

Las personas entrevistadas viven en el país y fueron entrevistados independientemente de su situación legal, lo que es un rasgo novedoso en las encuestas porque nos proporciona información sobre la situación regular o irregular sobre los inmigrantes, pero también sobre el contrato de trabajo regular o irregular. Además, la ESE proporciona un amplio conjunto de indicadores sobre valores, ideología, actitudes, situación laboral e ingresos económicos. El referente actitudinal y de comportamiento social y político está presente en todos los datos sobre cada país, lo que permite la comparación entre ellos (Mota, 2005). 
Hemos seleccionado 17 países $^{2}$ de acuerdo a los modelos de Estados de Bienestar con la finalidad de explicar los factores contextuales que influyen en las actitudes hacia la inmigración (Esping-Andersen, 2000; Morissen, 2008; Menz, 2008). El universo está formado por todas las personas de más de 15 años residentes en hogares privados, sin importar su nacionalidad, ciudadanía, idioma y estatus legal.

Por lo que respecta al concepto de "incertidumbre socioeconómica", la ESE nos permite captar el conocimiento inseguro o dudoso del estatus socio-laboral de los individuos en relación al empleo, al desempleo, al trabajo a tiempo parcial o sin contrato de los individuos y en general a la percepción futura de renta. La incertidumbre tiene bases objetivas o materiales, como son la posición contractual en el mercado de trabajo, relacionada con el riesgo, con la competencia por los recursos escasos de empleo y bienestar.

\subsection{Variables independientes}

En primer lugar, se incluye en el análisis un grupo de variables que nos informan sobre la posición socio-laboral individual y su situación socio-económica, como son el sexo, nivel de cualificación y edad, el contrato de trabajo, el empleo temporal, el empleo irregular, el desempleo, la búsqueda activa de empleo, la percepción subjetiva de los individuos sobre sus ingresos económicos (clasificados en tres categorías: "confortables, suficientes y muy difíciles"), los ingresos económicos en quintiles y la "fuente principal de ingresos en el hogar" (salarios, pensiones por jubilación, pensiones por desempleo y otros subsidios). Es decir, se tratan de variables que nos permite identificar la posición de los individuos en el mercado de trabajo y su estatus socio-económico, como ya se han utilizado en otros estudios (Card et al. 2005; Mayda, 2006, Ortega; Polavieja, 2009, entre otros).

En segundo lugar, analizamos variables de carácter individual que recoge el posicionamiento político entre la izquierda y la derecha: “ ¿Cual es su posicionamiento político en una escala de 0 a 10?". Esta pregunta la hemos transformado en tres categorías: izquierda, centro, derecha. Además incluimos otras variables referidas a valores con significado político relacionado con valores colectivos como el compromiso igualitario, la equidad y la justicia social (Schwartz, 2007). Estas variables son: “¿Los servicios sociales previenen contra la pobreza?”; ¿El gobierno debe reducir las desigualdades sociales?”; “¿El gobierno debe reducir impuestos?” y “¿Los servicios sociales lastran a la economía?". Estas variables relacionadas con el posicionamiento político contribuyen a explicar las diferencias de las actitudes, particular-

${ }^{2}$ Los países del modelo socialdemócrata Suecia, Noruega, Dinamarca y Finlandia; del modelo contributivo continental son Alemania, Bélgica y Holanda; del modelo mediterráneo Francia, España, Portugal y Grecia y de Europa del Este Polonia, Chequia, Hungría y Estonia. No hemos incluido los casos de Italia, Irlanda y Austria porque no figuran en la ESE (2008). 
mente entre la izquierda y la derecha política (Citrin et al. 2005; Saxton; Benson, 2003).

$\mathrm{Y}$, en tercer lugar, incluimos otras variables independientes externas concernientes al contexto, tales como la tasa de desempleo, la tasa de inmigración, el nivel de desigualdad (Índice Gini), riesgo de pobreza después de transferencias sociales y el PIB con la finalidad de explicar las diferencias entre los países estudiados. Estas variables proceden de otra fuente, Eurostat, y no figuran en la muestra de la ESE.

\subsection{Variable dependiente}

Con respecto a la variable dependiente, la ESE nos proporciona un grupo de preguntas que ilustran distintos grados de aceptación y de rechazo de la inmigración según el origen, la etnia y la raza. La variable dependiente que hemos tomado es: "Permitiría usted la entrada de muchos/algunos/pocos/ningunos inmigrantes procedentes de países pobres de fuera de Europa" y la comparamos con otras dos preguntas similares:"¿Permitiría usted la entrada de inmigrantes de la misma raza/grupo étnico? y “PPermitiría usted la entrada de inmigrantes de distinta raza/grupo étnico?". Las respuestas se clasifican en cuatro categorías: "muchos, algunos, pocos y ninguno".

Los inmigrantes de la misma raza/grupo étnico son los que tienen una mayor aceptación de acuerdo con la idea de "deseabilidad social" (Cea D'Ancona, 2002; 2009). Por el contrario, quienes tienen una menor "deseabilidad social" son los "inmigrantes procedentes de países pobres de fuera de Europa." No son "deseables socialmente" porque el hecho de ser "pobre" es un estigma social y connota probablemente mayor competencia por los recursos de empleo y bienestar. Además el hecho de ser "fuera de Europa" connota otro estigma: que son probablemente de "otra raza o grupo étnico", es decir físicamente diferentes. Para el análisis de regresión logística hemos recodificado la variable dependiente como una variable dicotómica: actitud liberal (muchos y algunos) y actitud restrictiva (pocos y ningunos), como ya se han hecho también en otros estudios porque permite ganar en significatividad en los resultados del análisis estadístico a tenor del número de casos (Card, et al 2005; Ceobanu; Escandell, 2010).

\section{Descripción, tendencias y factores influyentes en las actitudes}

En la tabla 1 las medias en relación a la escala sobre las actitudes hacia la inmigración ponen de manifiesto dos cuestiones: Primero, una tendencia hacia el aumento de las actitudes restrictivas ante la entrada de inmigrantes procedentes de países pobres de fuera de Europa entre los años 2002 y 2010 . $^{3}$ Segundo, la tenden-

${ }^{3}$ Por otra parte, la ESE ofrece dos variables que permiten estudiar la actitud ante los permisos de entrada de nueva inmigración diferenciando por su origen. La primera variable 
cia restrictiva se observa con más intensidad en aquellos países más afectados por la crisis, el desempleo y con altas tasas de inmigración, como son los casos de Grecia, Portugal, Hungría, República Checa y Reino Unido, todos ellos por encima de la media en el año 2010. En estos casos podría explicarse dicha tendencia por el aumento de la competencia por los escasos recursos de empleo y bienestar. Esta observación es similar a lo que han encontrado otras investigaciones (Citrin et al. 2005; Saxton; Benson, 2003; Ceobanu; Escandell, 2010, entre otros). Por el contrario, los países escandinavos, tienen actitudes más liberales y tolerantes hacia la inmigración -a excepción de Finlandia- lo que a priori podemos pensar que está relacionado con menores tasas de desempleo e inmigración, así como con un Estado del bienestar más generoso que reduce las desigualdades sociales y la competencia por los recursos de empleo y bienestar.

se refiere a la actitud ante los inmigrantes procedentes de países pobres de fuera de Europa. Esta variable conecta directamente con la hipótesis de la competencia socio-económica entre nativos e inmigrantes. $\mathrm{Y}$ tal y como se ha mencionado anteriormente, esperábamos que la crisis económica afectara a las actitudes ante la admisión de inmigrantes. Y, la segunda variable se refiere al permiso de admisión de inmigrantes de la "misma raza y grupo étnico" (lo que también nos permite mesurar el racismo) nos muestra una actitud más favorable hacia la admisión de aquellos inmigrantes de la misma raza.

En otras palabras, como señala Cea D’Ancona (2009:20-21), la comparación de estas dos variables nos permite ver la "deseabilidad social" respecto a la distinta procedencia, razas y grupos étnicos socialmente admisibles. Por consiguiente nótese que los inmigrantes de la "misma raza o grupo étnico" son más aceptados (media 2,20) y generan menor rechazo que los "inmigrantes procedentes de países pobres de fuera de Europa" (media 2,65). En este sentido Rodriguez Monter (2009) observa que la dimensión que generan menos "deseabilidad social” es el aspecto físico (“diferente raza y, además, de países pobres”). 


\begin{tabular}{|c|c|c|c|c|}
\hline \multicolumn{5}{|c|}{$\begin{array}{l}\text { Tabla } 1 \\
\text { Actitud hacia la inmigración. “¿Permitiría usted la entrada de inmigrantes } \\
\text { de países pobres de fuera de Europa?”: 1=Muy liberal (muchos); 4=Muy } \\
\text { restrictiva (ninguno) }\end{array}$} \\
\hline & & \multicolumn{3}{|c|}{ Actitud de la Población } \\
\hline & País & 2002 & 2008 & 2010 \\
\hline \multirow[b]{4}{*}{ Model Bismarkiano } & Bélgica & 2,25 & 2,40 & 2,53 \\
\hline & Alemania & 2,12 & 2,32 & 2,4 \\
\hline & Holanda & 2,35 & 2,42 & 2,5 \\
\hline & Media & 2,23 & 2,37 & 2,48 \\
\hline \multirow[b]{5}{*}{ Social-demócrata } & Noruega & 2,11 & 2,27 & 2,29 \\
\hline & Suecia & 1,82 & 1,82 & 1,81 \\
\hline & Dinamarca & 2,05 & 2,53 & 2,5 \\
\hline & Finlandia & 2,31 & 2,64 & 2,82 \\
\hline & Media & 2,04 & 2,32 & 2,36 \\
\hline \multirow[b]{3}{*}{ Liberal } & Reino Unido & 2,34 & 2,59 & 2,73 \\
\hline & Irlanda & 2,03 & - & 2,61 \\
\hline & Media & 2,15 & - & 2,67 \\
\hline \multirow[b]{5}{*}{ Mediterráneo } & Francia & 2,33 & 2,52 & 2,57 \\
\hline & España & 2,31 & 2,62 & 2,49 \\
\hline & Portugal & 2,67 & 2,84 & 2,81 \\
\hline & Grecia & 2,76 & 3,16 & 3,26 \\
\hline & Media & 2,7 & 2,79 & 2,78 \\
\hline \multirow{7}{*}{ Este Europa } & Polonia & 2,23 & 2,09 & 2,13 \\
\hline & Chequia & 2,43 & 2,81 & 2,89 \\
\hline & Hungría & 2,38 & 3,23 & 3,19 \\
\hline & Eslovenia & 2,3 & 2,51 & 2,5 \\
\hline & Media & 2,36 & 2,66 & 2,67 \\
\hline & Total & 2,24 & 2,59 & 2,65 \\
\hline & & 32805 & 34470 & 39723 \\
\hline
\end{tabular}

Fuente: Elaboración propia ESE (2002; 2008 y 2010)

El análisis de correspondencias múltiples, realizado con la ESE 2010, nos permite establecer una clasificación de los países de acuerdo a un grupo de categorías relacionadas con la percepción de incertidumbre económica (percepción subjetiva 
del nivel de ingresos; nivel de ingresos en quintiles; tipo contrato laboral; situación laboral -desempleado, pensionista, asalariado-) y las actitudes hacia la inmigración. La varianza explicada es del 65\% y el principal eje de clasificación es el horizontal (incertidumbre versus seguridad, $38 \%$ de la varianza). El segundo eje (vertical, explica el $28 \%$ de la varianza) permite clasificar las actitudes entre restrictivas y liberales.

\section{Gráfico 1}

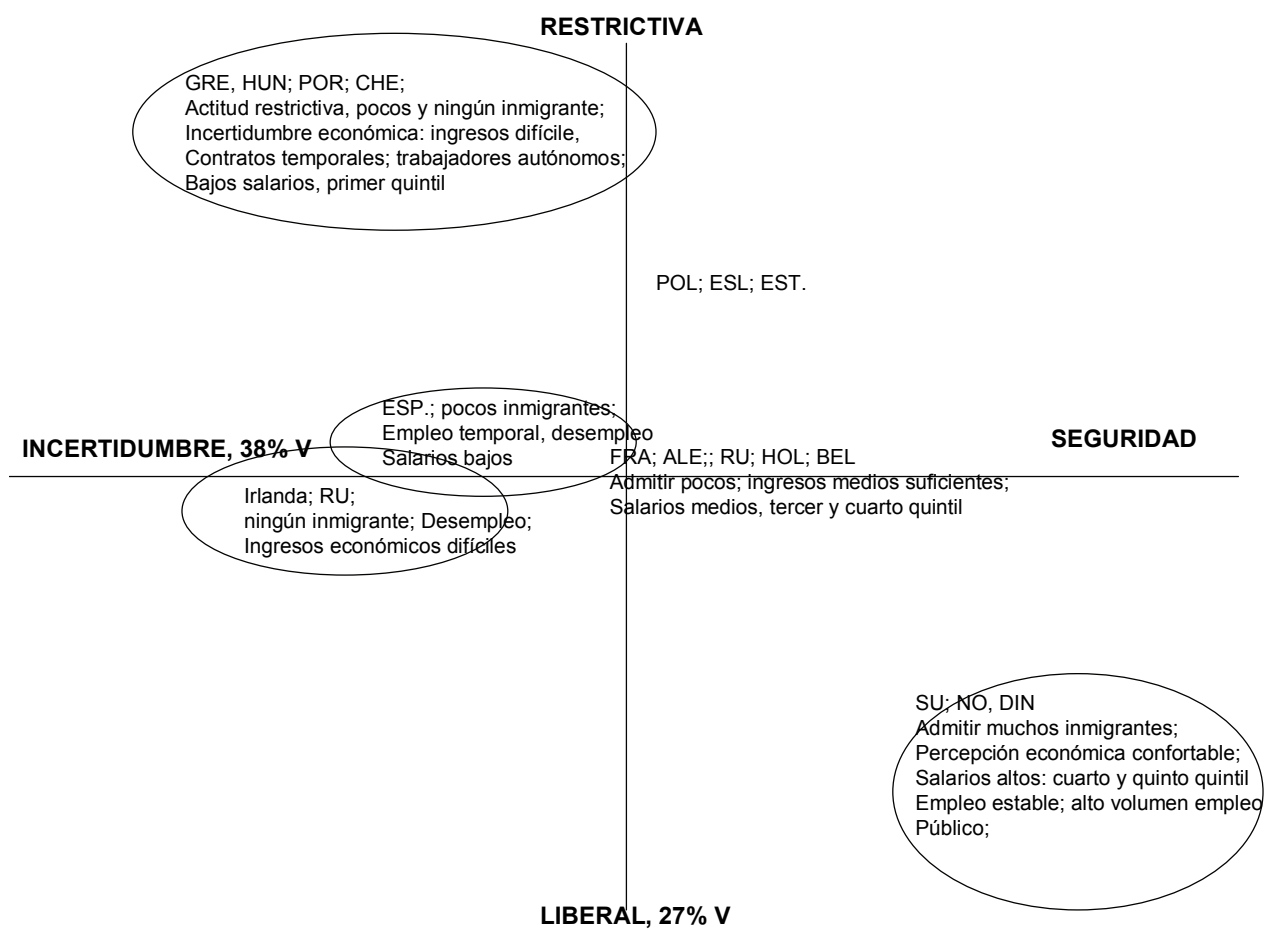

Fuente: Elaboración propia con datos ESE 2010.

En el gráfico 1 se observa la polarización de las actitudes. Por un lado, en el primer cuadrante se agrupan aquellos países que tienen mayor incertidumbre y actitudes restrictivas porque los ciudadanos perciben sus ingresos económicos como muy difíciles, hay mayor proporción de empleos temporales y de trabajadores autónomos. Estos países son Grecia, Hungría, Portugal y Chequia, con Estados del Bienestar débiles y bajo nivel de riqueza. Por otro lado, en el otro extremo situado en el cuarto cuadrante, se agrupan los países en los cuales los ciudadanos perciben sus ingresos económicos como confortables, salarios altos, empleo estable y tienen actitudes tolerantes y liberales hacia la entrada de inmigrantes. En este grupo se 
clasifican los países del Modelo de Bienestar Social-Demócrata Escandinavo (Suecia, Noruega y Dinamarca).

\subsection{Incertidumbre socio-económica}

En relación a la primera hipótesis (H1) podemos apuntar que las variables socioeconómicas están asociadas a las actitudes restrictivas ante la inmigración. En este sentido el análisis bivariado nos muestra que la percepción de incertidumbre en los ingresos económicos está relacionada con las actitudes restrictivas. ${ }^{4}$ En efecto, en los países estudiados la distribución de las actitudes según el nivel de renta presenta diferencias: aquellos individuos que tienen dificultades y sobre todo los que tienen "muchas dificultades económicas" son los más partidarios de una política restrictiva en la concesión de permisos de admisión de inmigrantes. Estos señalan "pocos" o incluso "ningún" inmigrante procedentes de "países pobres no europeos". Esta tendencia restrictiva aumenta en la encuesta de 2010. En el conjunto de los países estudiados, la mayoría de quienes tienen muchas dificultades en los ingresos económicos en el hogar son los que rechazan más claramente a la inmigración. Mientras que en el otro extremo, los que tienen "ingresos económicos confortables" en el hogar están en su mayoría a favor de la admisión de "algunos e incluso muchos inmigrantes" (tabla 2). En pocas palabras, podemos observar una posición muy restrictiva en la entrada cuando se trata de inmigrantes con el estigma de pobres. ${ }^{5}$

\section{Tabla 2}

Percepción de renta familiar y actitudes frente a la admisión de inmigrantes procedentes de países pobres de fuera de Europa. en \%.

\begin{tabular}{|c|c|c|c|c|c|c|c|c|c|}
\hline & \multicolumn{2}{|c|}{ Muchos } & \multicolumn{2}{|c|}{ Algunos } & \multicolumn{2}{|c|}{ Pocos } & \multicolumn{2}{|c|}{ Ninguno } & \multirow[b]{2}{*}{ Total } \\
\hline & 2008 & 2010 & 2008 & 2010 & 2008 & 2010 & 2008 & 2010 & \\
\hline Confortable & 16,7 & 15 & 46,9 & 41,1 & 29,5 & 31,7 & 6,9 & 12,3 & 100 \\
\hline Suficiente & 12 & 10,4 & 38,9 & 36,5 & 35,7 & 36,5 & 13,4 & 19,4 & 100 \\
\hline Difícil & 10,5 & 10,7 & 31,2 & 34,5 & 37,5 & 34,5 & 20,9 & 26,8 & 100 \\
\hline Muy difícil & 12,7 & 12,5 & 22,5 & 28 & 33,6 & 30,6 & 31,2 & 35,6 & 100 \\
\hline $\mathrm{N}(2008)$ & \multicolumn{9}{|c|}{34470, V Cramer , 136} \\
\hline $\mathrm{N}(2010)$ & \multicolumn{9}{|c|}{ 39723, V Cramer ,114 } \\
\hline
\end{tabular}

Fuente: Elaboración propia basada en ESE (2008 y 2010), 17 países europeos

${ }^{4}$ En efecto, hay una relación lineal entre la percepción subjetiva de los ingresos económicos y actitudes, por ejemplo, para el año 2008 hemos hallado que el coeficiente de determinación del análisis bivariado entre "ingresos económicos muy difíciles" y la actitud negativa hacia la entrada de "ningún inmigrante" es fuerte $\left(\mathrm{R}^{2} 0,587\right)$.

${ }^{5}$ Un estudio de Simón y Sikich (2007) sobre las actitudes hacia la inmigración pone de relieve una demanda para "reducir mucho" el número de de inmigrantes en Francia, Alemania y Reino Unido. Esta es la opinión mayoritaria entre los encuestados. 


\subsection{Influencia factores individuales}

Los factores socioeconómicos influyentes en las actitudes se recogen en la regresión logística binomial de la tabla 3 . Hemos utilizado en la regresión los datos de la encuesta de 2008 porque ofrece las variables ideológicas y de compromiso igualitario útiles para nuestro propósito y que no figuran en la encuesta de 2010. No obstante, cuando las variables son parecidas para ambos años los resultados nos vuelven a confirmar parecidos grados de influencia. Los resultados se explican a partir de la estimación de dos modelos. En el modelo 1 se incluyen las variables de carácter individual, así como las relativas a la posición socio-económica objetiva. En este sentido el género es una variable no significativa. Por grupos de edad se pone de relieve que a medida que aumenta ésta aumenta el peso de las actitudes restrictivas hacia la inmigración, de modo que los más jóvenes tienen actitudes más liberales. Los mayores de más de 54 años tienen actitudes más restrictivas, lo que nos indica una relación lineal y concuerda con lo hallado en otros estudios (véase Solé et al. 2000, entre otros). El nivel de estudios parece jugar un papel importante en las actitudes: quienes tienen estudios primarios tienen actitudes más restrictivas que aquellos que tienen estudios universitarios. Este resultado coincide con los hallados por Hainmueller y Hiscox (2007), quienes señalan que las personas con mayor nivel de educación tienen otros valores sobre la tolerancia y la admisión de la diferencia o bien están más o menos expuesto a la competencia en el mercado de trabajo según el nivel de cualificación de los individuos (véase Ortega y Polavieja, 2009). Además, aquí se puede ver de nuevo una relación lineal y el efecto competencia: aquellos con menores niveles de estudios son los principales partidarios de limitar la entrada de inmigrantes (Kogan, 2003; Saxton; Benson, 2003; Card, et al. 2005).

\subsection{Influencia factores socio-económicos}

Las variables relacionadas con el hecho de haber estado "desempleado en los últimos cinco años" y con el "desempleo actual" no son significativas en relación a la actitud de rechazo a la entrada de inmigrantes, lo que puede estar relacionado con el efecto composición (ya que hay muchos inmigrantes que han estado en situación de desempleo) y con el número de casos de desempleados existentes en la muestra. ${ }^{7}$

\footnotetext{
${ }^{6}$ Las actitudes favorables de los ciudadanos hacia la entrada de los inmigrantes dependen también del nivel de nivel de cualificación de éstos últimos. Lamentablemente la ESE de los años 2002 y 2008 no contiene ninguna pregunta relacionada con ello. Pero si la encuesta del año 2001 estudiada por Card et al. (2005). Estos autores concluyen que los inmigrantes mejor aceptados son aquellos que tienen cualificaciones profesionales, estudios altos y habilidades lingüísticas. También Hainmueller y Hiscox (2007) y Mau Burkhardt (2009) concluyen en parecidos términos analizados a partir de los datos de la ESE del año 2003.

${ }^{7}$ La relación entre la tasa de desempleo y las actitudes restrictivas pone de manifiesto que los países con mayor desempleo son aquellos que tienen actitudes más restrictivas
} 
Los trabajadores con contratos temporales, y particularmente aquellos que trabajan irregularmente sin contrato son los que tienen más peso en las actitudes restrictivas hacia la inmigración. En otras palabras, son los trabajadores irregulares quienes más perciben la competencia con los inmigrantes por los puestos de trabajo. El hecho de trabajar "sin contrato laboral" es ya de por si mismo un indicador de incertidumbre; por consiguiente no debe extrañar que sean precisamente éstos trabajadores quienes más rechacen a la inmigración en tanto que comporta más competencia, como ha demostrado Reyneri (2006) para el caso italiano y Cea D'Ancona; Vallés (2011), para el caso español.

Quienes perciben que sus ingresos económicos son difíciles, con incertidumbre, tienen más probabilidades de tener actitudes hostiles y restrictivas hacia la entrada de inmigrantes, que aquellos que tienen ingresos confortables. En pocas palabras, las variables socio-económicas tienen capacidad predictiva sobre las actitudes ante la inmigración, lo que contrasta con lo hallado por Rodríguez Monter (2009:224), quién ha encontrado más importante el papel predictivo de las variables culturales. Pero coincidimos con dicha autora en la importancia que tienen algunas variables culturales, como es el caso de la ideología política.

(Lituania, Finlandia, Portugal, España y Grecia) y, por el contrario, los países con menores tasas de desempleo tienen actitudes menos restrictivas $\left(\mathrm{R}^{2}: 0.345\right)$. En otras palabras, la tasa de desempleo contribuye explicar la competencia en el mercado de trabajo y su efecto en las actitudes negativas hacia la inmigración. 


\begin{tabular}{|c|c|c|c|c|c|c|c|}
\hline \multicolumn{8}{|c|}{$\begin{array}{l}\text { Tabla } 3 \\
\text { Actitudes hacia la inmigración. } 0=\text { Liberal; } 1=\text { Restrictiva }\end{array}$} \\
\hline & & \multicolumn{3}{|c|}{$\begin{array}{l}\text { MODELO 1. VARIABLES } \\
\text { INDIVIDUALES Y SOCIO } \\
\text { ECONÓMICAS }\end{array}$} & \multicolumn{3}{|c|}{$\begin{array}{l}\text { MODELO } 2 . \\
\text { IDEOLOLOGÍA Y } \\
\text { COMPORMISO } \\
\text { IGUALITARIO }\end{array}$} \\
\hline & & $\mathrm{B}$ & E.T. & & $\mathrm{B}$ & E.T. & $\operatorname{Exp}(B)$ \\
\hline \multirow{10}{*}{$\begin{array}{l}\text { Variables } \\
\text { Individuales }\end{array}$} & & & & $\operatorname{Exp}(\mathrm{B})$ & & & \\
\hline & $\begin{array}{l}\text { Género, } \\
\text { Ref=Hombre }\end{array}$ & $-0,101$ & 0,055 & 0,904 & $-0,114 * *$ & 0,061 & 0,892 \\
\hline & $\begin{array}{l}\text { Ref=Edad, 16-24 } \\
\text { años }\end{array}$ & & & & & & \\
\hline & 24-35 años & $-0,318 * *$ & 0,143 & 0,728 & $-0,49 * *$ & 0,168 & 0,613 \\
\hline & 35-44 años & $-0,381 * * *$ & 0,088 & 0,683 & $-0,446 * * *$ & 0,101 & 0,64 \\
\hline & 45-54 años & $-0,206^{* *}$ & 0,078 & 0,814 & $-0,29 * * *$ & 0,089 & 0,748 \\
\hline & Más 54 años & $-0,193 * *$ & 0,078 & 0,825 & $-0,208^{*}$ & 0,086 & 0,812 \\
\hline & $\begin{array}{l}\text { Ref=Estudios } \\
\text { primarios }\end{array}$ & & & & & & \\
\hline & $\begin{array}{l}\text { Estudios secunda- } \\
\text { rios }\end{array}$ & $1,137 * * *$ & 0,11 & 3,117 & $1,11 * * *$ & 0,127 & 3,035 \\
\hline & $\begin{array}{l}\text { Estudios Univer- } \\
\text { sitarios }\end{array}$ & $0,699 * * *$ & 0,071 & 2,012 & $0,628 * * *$ & 0,077 & 1,875 \\
\hline \multirow[t]{8}{*}{$\begin{array}{l}\text { Variables } \\
\text { socio- } \\
\text { económicas }\end{array}$} & \begin{tabular}{|l} 
Incertidumbre \\
en la trayectoria \\
(Alguna vez \\
desempleado en \\
los últimos 5 \\
años)
\end{tabular} & $-0,016$ & 0,063 & 0,984 & $-0,07$ & 0,069 & 0,932 \\
\hline & Desempleado & 0,044 & 0,094 & 1,045 & 0,01 & 0,107 & 1,01 \\
\hline & $\begin{array}{l}\text { Ref }=\text { Contrato } \\
\text { permanente }\end{array}$ & & & & & & \\
\hline & Contrato temporal & $-0,091$ & 0,107 & 0,913 & $-0,142$ & 0,124 & 0,867 \\
\hline & $\begin{array}{l}\text { Empleado sin } \\
\text { contrato legal }\end{array}$ & $-0,154$ & 0,114 & 0,858 & $-0,185^{*}$ & 0,132 & 0,831 \\
\hline & $\begin{array}{l}\text { Ref=Ingresos } \\
\text { económicos } \\
\text { confortables }\end{array}$ & & & & & & \\
\hline & $\begin{array}{l}\text { Ingresos suficien- } \\
\text { tes }\end{array}$ & $-0,472 * * *$ & 0,086 & 0,624 & $-0,353 * * *$ & 0,097 & 0,702 \\
\hline & $\begin{array}{l}\text { Ingresos econó- } \\
\text { micos difíciles }\end{array}$ & $-0,196 * *$ & 0,067 & 0,822 & $-0,159 *$ & 0,077 & 0,853 \\
\hline
\end{tabular}




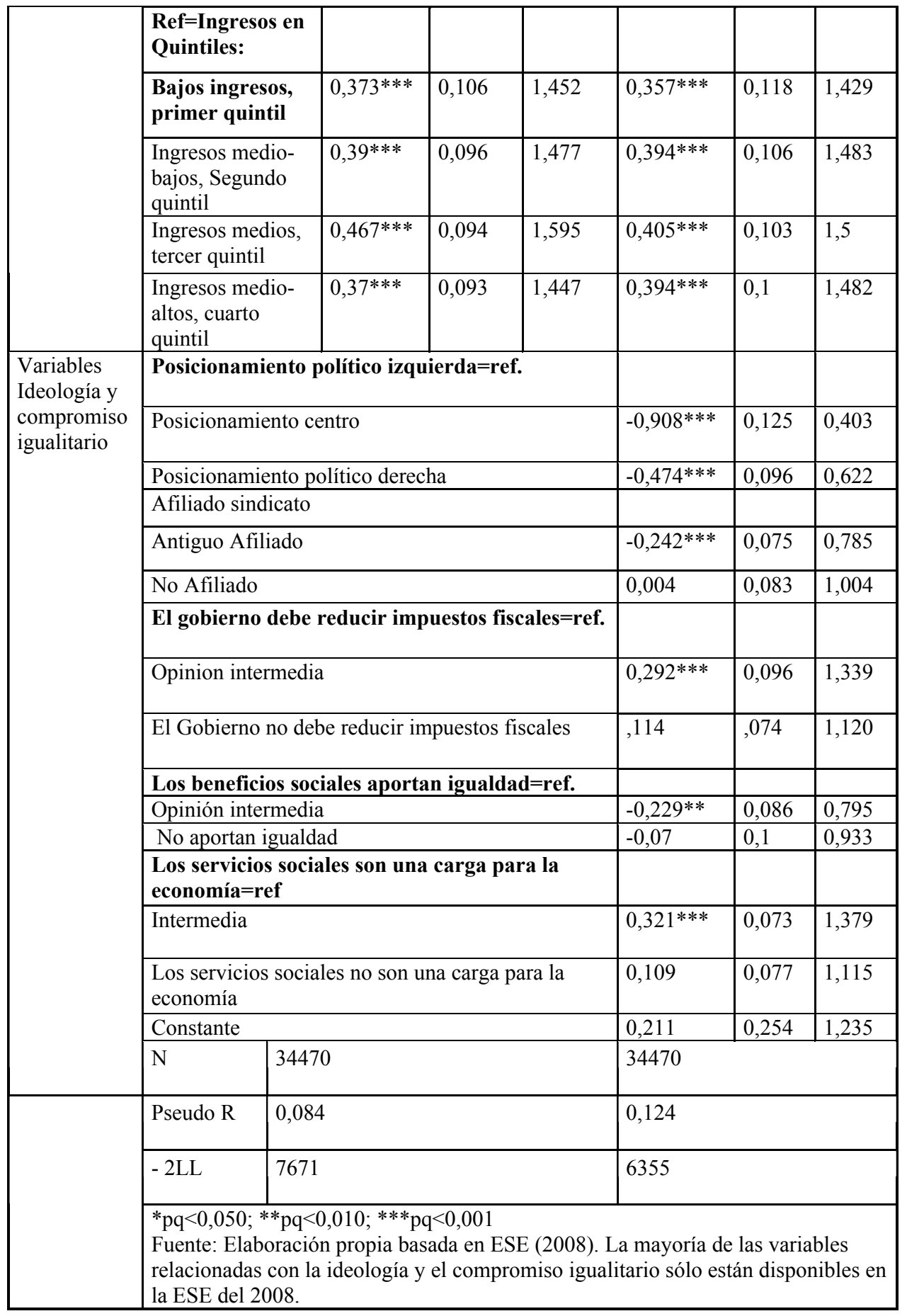




\subsection{Posicionamiento ideológico izquierda-derecha política, actitudes y valores}

En relación a la segunda hipótesis (H2) referida el papel que juega los valores ideológicos, el compromiso igualitario ${ }^{8}$ y el posicionamiento izquierda-derecha presuponemos que tiene un efecto importante en las actitudes restrictivas. En principio, como era de esperar, quienes se identifican ideológicamente con posiciones de izquierda tienen más probabilidades de tener actitudes más liberales y tolerantes hacia la inmigración que aquellos que se identifican con la derecha política, lo que se explica porque los primeros comporten más los valores del compromiso igualitario y colectivo que los segundos. Además, esta variable tiene un notable peso sobre las actitudes. A ello hay que añadir que entre 2002 y 2010 se ha incrementado bastante la actitud restrictiva de aquellos que se declaran políticamente próximos a la derecha (véase tabla anexa $1^{\mathrm{a}}$ ). Los países donde se registra una correlación positiva y significativa entre ideología política y actitudes, son Alemania $^{9}(, 226)$, Dinamarca (,317), Francia (282), Noruega (,247) y Holanda (,173). En estos países el peso de la extrema derecha es importante e influye en el debate sobre el rechazo a la inmigración. ${ }^{10}$

${ }^{8}$ Los afiliados sindicales tienen una actitud también restrictiva hacia la inmigración, por ejemplo en el año 2010 la media en la mencionada escala de 1 a 4 fue de 2,49, por debajo del 2,65 general, es decir por debajo de dicha media.

${ }^{9}$ Por ejemplo, en Alemania el $43 \%$ de los que se declaran próximos al partido de extrema derecha NPD/DVU opinan que no debe permitirse la entrada de "ninguno" y el 57\% declaran que "pocos" inmigrantes. Por el contrario, los que se posicionan más a la izquierda son más liberales: el 35\% de los votantes del Partido Verde son partidarios de la admisión de "mucha" inmigración, seguido del $22 \%$ de los votantes del partido socialdemócrata SPD, ambos partidos de izquierda están por encima de la media alemana (13\%).

En el caso de Dinamarca el 19\% de los votantes el partido nacionalista Danks Folkerparti declara la entrada de "ningún inmigrantes". Y el 58\% de los votantes de este mismo partido son partidarios de la entrada de "pocos inmigrantes". Por el contrario, los votantes de izquierda, del Green Party son también los más partidarios (55\%) de la admisión de "mucha inmigración". La misma pauta se repite en Holanda: son contrarios a la entrada de inmigrantes el 39\% de los votantes de derechas, como son, del Social Reformed Party, el $30 \%$ de los votantes de List Pim Fortuyn y el 22\% del List Wilders, quienes declaran la entrada de "ningún inmigrante." Mientras que el 24\% de los votantes del Green Party son partidarios de la entrada de "muchos inmigrantes" (media nacional del 13\%). En Francia ocurre algo parecido, pero en menor proporción. Los partidarios de admitir mucha inmigración son los votantes de Les Verts (18\%), cuando la media es muy baja (9\%). Los partidarios de una política radical restringida (ningún) son los votantes del derechista Front National (27\%). A modo de resumen: en el conjunto de los países estudiados, el 36\% de quienes declaran que "ningún" inmigrante debería entrar en su país se posicionan en los partidos de extrema derecha, situados en el extremo de una la escala de 0 a 10.

${ }^{10}$ Por otra parte, en la tabla anexa 2a se muestra como las actitudes hacia la inmigración se relacionan con el posicionamiento político sobre el compromiso igualitario, la justicia 
En pocas palabras, el posicionamiento político izquierda-derecha es una variable explicativa de las actitudes hacia la inmigración; lo que es mucho más evidente cuando hablamos de partidos de extrema derecha, cuyo discurso está fundamentalmente centrado en una valoración negativa de la inmigración, en valores antiuniversalistas como ha puesto de relieve Innerarity y Acha (2010). Asimismo, los afiliados a los sindicatos ${ }^{11} \mathrm{y}$ los antiguos afiliados tienen actitudes menos restrictivas que los no afiliados y sus valores ideológicos están más cerca del compromiso igualitario que la de otros grupos sociales. De hecho la posición política de los afiliados se sitúa en el espacio de centro-izquierda.

Las otras variables relacionadas con algunos valores demostrativos del "compromiso igualitario" (Schwartz, 2007), nos muestra como aquellos que opinan que los servicios sociales previenen la pobreza y los que tienen una posición intermedia tiene una actitud más liberal y tolerante que aquellos que opinan que los servicios sociales no previenen, lo que concuerda con el principio moral del igualitarismo. La opinión de que el "gobierno debe reducir las desigualdades" no es significativa en la determinación de las actitudes. Por el contrario, aquellos que tienen más probabilidades de tener peso en la determinación de las actitudes restrictivas son quienes sostienen que "los servicios sociales constituyen una carga para la economía" y quienes contestan que el "gobierno debe reducir los impuestos". En ambos casos estas dos posiciones están relacionadas con posicionamientos políticos de derecha,

social y la orientación de la acción de gobierno en materia de servicios sociales (Estas variables sólo figuran en la ESE de 2008). En efecto, según la valoración de las políticas sociales hay diferencias en las actitudes entre quienes se posicionan a la izquierda y a la derecha. Por un lado, para aquellos que se posicionan a la izquierda, una ligera mayoría de ellos tienen actitudes favorables (liberales) hacia la inmigración y opinan que los "beneficios sociales previenen contra la pobreza" y que el "gobierno debe reducir las desigualdades"; lo que concuerda con los valores del igualitarismo, incluso con la inmigración procedente de países pobres. En este sentido son más inclusivos y tienen valores más colectivistas que aquellos individuos que se declaran de derechas. Por otro lado, quienes se posicionan en la derecha consideran en mayor proporción que los "beneficios sociales constituyen una carga para la economía" y que el "gobierno debe reducir impuestos". En general, parece que éstos tienen una actitud más restrictiva y tienen posiblemente valores más individualistas que los de izquierda, lo que concuerda en cierto modo con lo hallado por Cea D'Ancona y Vallés, (2010) para el caso español. Pero a pesar de las diferencias indicadas entre izquierda y derecha, en general podríamos pensar que probablemente los valores del igualitarismo son más difíciles de promover cuando la inmigración tiene características raciales, étnicas y culturales muy distintas a la europea (Rodiguez Monter, 2009), o como señalaba Habermas (2000) la heterogeneidad étnica y racial dificulta la construcción de lazos de solidaridad.

${ }^{11}$ Las medias de las respuestas de los afiliados a la pregunta sobre si estaría dispuesto a permitir la entrada de inmigrantes de países pobres de fuera de Europa está por debajo de la media en el año 2010, dicha media fue de 2,65 mientras la de los afiliados fue de 2,49 
lo que concuerda con actitudes conservadoras y con la observación de Saxton y Benson (2003) sobre ideología y actitudes hacia la inmigración.

\subsection{Efecto país e influencia de las variables contextuales sobre las actitudes}

En relación a la tercera hipótesis $(\mathrm{H} 3)$, hemos hallado que el coeficiente de determinación es más importante para las actitudes restrictivas $\left(\mathrm{R}^{2} 0,410\right)$ que para las actitudes liberales. En otras palabras, las variables contextuales tienen mayor capacidad explicativa sobre las actitudes restrictivas que sobre las actitudes liberales $\left(\mathrm{R}^{2}\right.$ $0,130)$. Las variables contextuales que tienen más peso para explicar las actitudes restrictivas son, por este orden, el índice de desigualdad de Gini (beta ,755), la tasa de desempleo (beta ,455) y el PIB per cápita (beta -,139), como se pone de relieve en los coeficientes del ejercicio de regresión lineal que hemos realizado. En efecto, el la desigualdad en los ingresos económicos tiene una relación de influencia notorio en las actitudes restrictivas, de modo que podemos decir que en los países con mayores desigualdades, como son Lituania, Grecia, Portugal y España, las actitudes de los ciudadanos hacia la inmigración son más restrictivas que en aquellos países más igualitaristas, como son Suecia, Noruega, Dinamarca. El riesgo de pobreza también tiene una influencia positiva y significativa sobre las actitudes restrictivas, de manera que en aquellos países donde el riesgo de pobreza es mayor, también es mayor la probabilidad de tener una actitud restrictiva hacia la inmigración. La tasa de desempleo tienen una influencia negativa con las actitudes restrictivas, lo que interpretamos como que en aquellos países donde las tasas de desempleo son menores también tienden a ser menores las probabilidades de las actitudes restrictivas hacia la inmigración. Y el PIB tiene una relación de determinación negativa con las actitudes restrictivas, lo que significaría que cuanto mayor es el PIB per cápita, es decir, mayor riqueza, más favorecería las actitudes tolerantes y liberales hacia la inmigración, como también ha demostrado Mayda (2006). Ejemplo de ello son los países escandinavos, donde el Estado del Bienestar generoso amortigua las desigualdades y la competencia por los recursos de empleo y bienestar. En ellos el "compromiso igualitario" con los inmigrantes correlaciona de forma positiva y significativa con el nivel de riqueza del país, con el gasto en protección social y con la generosidad de las prestaciones por desempleo de larga duración.

\section{Conclusiones y discusión}

En este artículo hemos contrastado el notable peso que tiene el nivel de estudios y el posicionamiento político izquierda-derecha en la determinación de las actitudes (prácticamente el doble que las otras variables); le siguen en importancia las otras variables socio-económicas que pesan sobre las actitudes negativas hacia la inmigración. En este sentido aportamos tres conclusiones siguiendo el hilo conductor de las hipótesis.

En primer lugar (H1), el análisis descriptivo de los datos sugiere que el cambio en el contexto económico ha producido un aumento de actitudes contrarias a la 
entrada de inmigrantes entre los años 2002 y 2010. Esta actitud varía entre países, siendo aquellos que tienen mayores tasas de desempleo y mayor desigualdad (índice de Gini) los que tienen actitudes más restrictivas hacia la entrada de inmigrantes. La competencia por los recursos de empleo y bienestar contribuye a explicar las actitudes. Hemos encontrado un efecto significativo de las variables ligadas a la situación socio-económica de los individuos. De este modo el nivel educativo, la edad, el contrato de trabajo, el nivel de ingresos económicos, así como la incertidumbre en los ingresos económicos, tienen un efecto estadísticamente significativo para explicar las actitudes hacia la inmigración. Este hallazgo relacionado con la importancia de las variables socio-económicas difiere de los resultados obtenidos por Rodriguez Monter (2009), quien por el contrario encuentra mayor capacidad predictiva en las otras variables culturales.

En segundo lugar (H2), observamos como la variable ligada al posicionamiento político izquierda-derecha juega un papel muy influyente a la hora de explicar las diferentes actitudes que los individuos adoptan hacia la admisión de inmigrantes. Esto sugiere que más allá de los cambios en el contexto económico, la posición político-ideológica del individuo es un factor notable para entender la actitud individual frente a la inmigración. Dado que este factor es en principio más constante a lo largo del tiempo, es decir, no está tan sujeto a variaciones cíclicas de la economía, su significatividad es todavía más interesante en el contexto de la actual crisis económica. El posicionamiento en la extrema derecha es el que marca más claramente las actitudes más restrictivas, anti-universalista y anti-igualitarias con quienes son "diferentes", como es el caso de los "inmigrantes procedentes de países pobres de fuera de Europa". Por el contrario, los valores asociados al compromiso igualitario (como son las opiniones que juegan los "servicios en la prevención de la pobreza" y la idea de que el "gobierno debe reducir las desigualdades") contribuyen a explicar las actitudes favorables y liberales hacia la inmigración, especialmente la de aquellos individuos que se posicionan en la izquierda política.

En tercer lugar (H3), las variables contextuales también tienen influencia sobre las actitudes. Particularmente las variables relacionadas con la desigualdad y el riesgo de pobreza contribuyen a la formación de las actitudes negativas hacia la inmigración. La desigualdad social (índice de Gini), el riesgo de pobreza y la tasa de desempleo son variables contextuales que contribuyen a explicar las actitudes restrictivas hacia la inmigración según países. Y, por el contrario, un PIB per cápita alto (un indicador de mayor riqueza) contribuye a generar actitudes más liberales hacia la inmigración porque reduce el efecto de competencia por los recursos de empleo y bienestar. En breve, las actitudes están condicionadas notablemente por el nivel de riqueza y la redistribución del bienestar. Pero también por las características de los inmigrantes, como el hecho de tener la "misma raza", proceder de países europeos, alto nivel de estudios y habilidades lingüísticas. La homogeneidad étnica y racial contribuye a facilitar la integración social de los inmigrantes. Por el contrario, la heterogeneidad dificulta la construcción del compromiso igualitario (Habermas, 2000). 


\subsection{Discusión}

El estudio de las actitudes hacia la inmigración es importante para conocer los problemas de la movilidad de los trabajadores de terceros países en la UE, como se ha manifestado con la aplicación de la Directiva Bolkestein. ${ }^{12}$ Las actitudes hostiles y restrictivas obstaculizan la movilidad geográfica y con ello la capacidad de ajuste y de flexibilidad de los mercados de trabajo, máxime cuando hoy sabemos que quienes más aportan movilidad son precisamente los inmigrantes de terceros países y no los trabajadores europeos, como se pensaba inicialmente con la construcción de un mercado único de trabajo.

La novedad de nuestros hallazgos en este estudio sugiere subrayar el poder explicativo de las variables ideológicas y las opiniones políticas en la conformación de las actitudes. La importancia de la ideología para atenuar el impacto de la competencia por los recursos económicos es un hallazgo importante para considerar el desarrollo de las políticas de integración: la producción de un discurso solidario es fundamental para construir una opinión pública tolerante y para luchar contra el racismo y la xenofobia. Además la edad y el nivel de estudios tienen una relación lineal con las actitudes. La politización de la inmigración como "problema" en la agenda política brinda hoy, al mismo tiempo, una oportunidad para el debate ideológico y la reconstrucción de la solidaridad, los derechos de ciudadanía y valores normativos de equidad social. Por todo ello es particularmente interesante el discurso sindical en términos de "compromiso igualitario", integración social y solidaridad de clase porque puede contribuir en la aceptabilidad social de la categoría del trabajador inmigrante.

El desarrollo de una política sindical a través de su inclusión en la agenda de la negociación colectiva podría contribuir al arraigo de actitudes tolerantes con la gestión de la diversidad. Por ello es importante la participación de los inmigrantes como representantes sindicales; su presencia en las mesas de negociación en las

12 En enero y febrero del 2009 los trabajadores de Lindsey Oil Refinery en Linconlshire (Reino Unido) llevaron a cabo una huelga contra la contratación de trabajadores italianos y portugueses que había realizado la empresa. Este rechazo a los trabajadores inmigrantes tiene importantes implicaciones. Primero, porque no se trata fundamentalmente de "inmigrantes", sino de trabajadores europeos que tiene "movilidad" en el espacio europeo como recoge la Directiva Bolkestein 76/71/EC. Segundo, porque estos trabajadores no están sujetos a la negociación colectiva del país de acogida. Tercero, porque implica una competencia por la rebaja de salarios mediante la movilidad de trabajadores de otros países y por tanto un riesgo de dumping social que socava el poder de los propios sindicatos y de la acción colectiva. Pero también la reacción hostil de los trabajadores nativos dificulta la construcción de un mercado de trabajo único europeo. Además dificulta aún más la aceptación y la solidaridad con los trabajadores extranjeros de otros trabajadores procedentes de países de fuera de Europa (Broughton, 2009). 
empresas; el establecimiento de un plan de acogida; la provisión de una formación básica profesional y lingüística; la traducción de textos, en especial aquellos que están relacionados con la prevención de riesgos laborales (Hernández, 2012); la adaptación de horarios y jornadas a la conciliación entre el tiempo de trabajo y la vida laboral, familiar y personal; la ampliación de los permisos para aquellos que tienen un origen lejano geográficamente; la organización de jornadas informativas desde los sindicatos y la promoción de actividades orientadas a la cohesión social y a la convivencia, entre otras (Jódar, et. al. 2011). En este sentido consideramos como un esfuerzo estimable el impulso - a partir del año 2000- de cuatro directivas de la Unión Europea contra la discriminación por razón de género, raza y etnia ante el temor del avance del nacionalismo de extrema derecha en muchos países europeos. Además, la UE ha recomendado el impulso de un código deontológico para los medios de comunicación con la finalidad de propiciar un tratamiento ecuánime sobre la inmigración.

Por todo ello, se sugiere como necesaria una política global europea para definir principios y normas comunes relativas a la coherencia y solidaridad entre los Estados miembros. Dicha política tendría que incluir estrategias para el empleo, subsidios para las situaciones de desempleo, mecanismos de lucha contra la pobreza y la desigualdad, políticas de integración social, políticas de protección social, políticas contra la discriminación étnica y racial, así como un discurso plural y multicultural destinado a la conformación de la opinión pública. Igualmente parece necesario reforzar la cooperación entre las administraciones públicas nacionales para mejorar la gobernanza multinivel en esta materia sensible. Finalmente el avance de una política común de inmigración tendría que concretarse mediante indicadores cuantificables con la finalidad de evaluar la acción emprendida.

Barcelona 15/03/2011, actualización datos ESE en 10 de mayo 2012. 


\section{Tablas anexas}

\begin{tabular}{|c|c|c|c|c|c|c|}
\hline \multicolumn{7}{|c|}{\begin{tabular}{|l|} 
Table 1A \\
Ideología política y actitud hacia la inmigración. en \% \\
\end{tabular}} \\
\hline & \multicolumn{2}{|r|}{2002} & \multicolumn{2}{|r|}{2008} & \multicolumn{2}{|r|}{2010} \\
\hline & LIBERAL & RESTRICTIVA & LIBERAL & RESTRICTIVA & LIBERAL & RESTRICTIVA \\
\hline Izquierda & 13,8 & 8,1 & 12,6 & 10,1 & 24,4 & 17,5 \\
\hline Centro & 73,9 & 79,6 & 74 & 69,9 & 53,2 & 53,1 \\
\hline Derecha & 12,3 & 12,3 & 13,4 & 20 & 22,4 & 29,4 \\
\hline Total & 100 & 100 & 100 & 100 & 100 & 100 \\
\hline $\mathrm{N}$ & & 32805 & & 34470 & 39723 & \\
\hline V Cramer & & 0,110 & & 0,091 & 0,102 & \\
\hline
\end{tabular}

Fuente: Elaboración propia basada en ESE (2002; 2008 y 2010), 17 países europeos

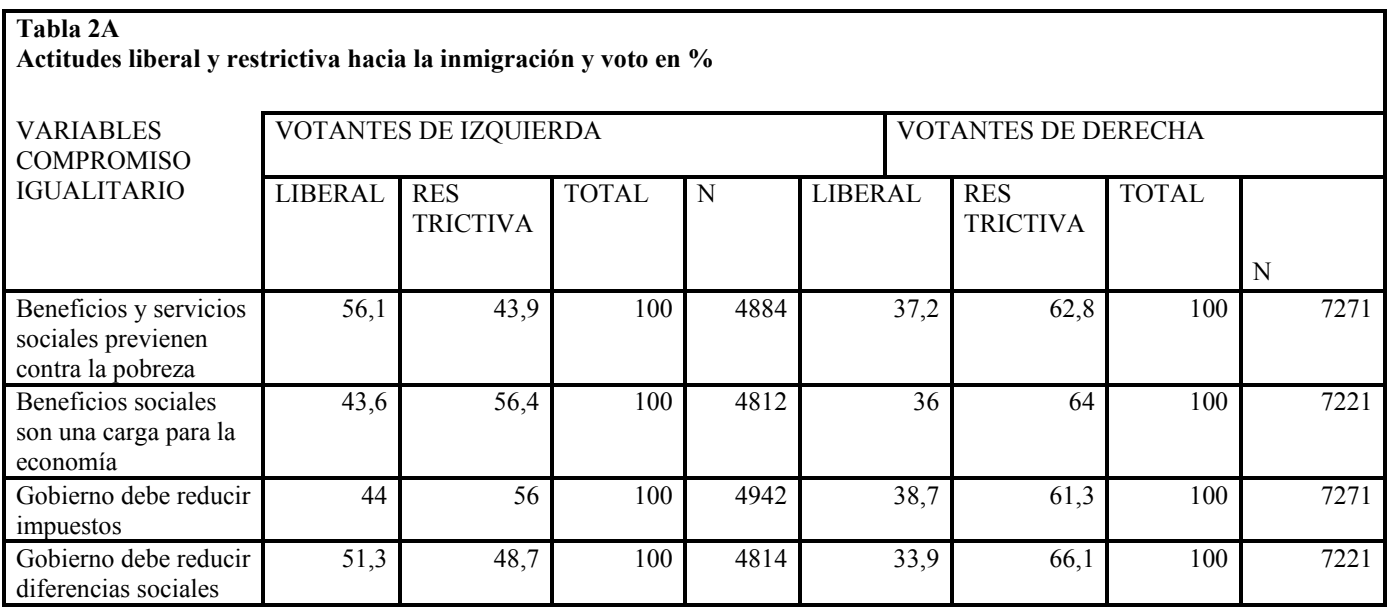

Fuente: Elaboración propia basada en ESE (2008), 17 países europeos. Estas variables sólo figuran en la ESE (2008).

\section{Bibliografía}

Alesina, A.; Glaser, E.L. (2004). Fighting Poverty in the US and Europe. A World of difference. Oxford University Press.

Álvaro Estramiana, J.L.; Rodriguez Monter, M. (2010). "Valores culturales y actitudinales hacia la inmigración en Europa." Revista Psicología, Conocimiento y Sociedad, http://revista@psico.edu.uy 
Balch, A. (2010). "Economic Migration and the Politics of Hospitality in Spain: Ideas and Policy Change", vol.38, n 5, pp-1037-1064

Beck, U. (1998). La sociedad del riesgo. Barcelona: Paidós.

Broughton, A. (2009). "Energy sector hit by styrikes over use foreing workers." Eironline, http.eurofound.europa.eu/eiro

Card, D.; Dustmann, C.; Prestón, I. (2005). ’Understanding attitudes to immigration: The migration and minority module of the first European Social Survey." Working Paper, $\mathrm{n}^{\circ}$ 5. London: Cream (Centre for Research and Analysis of Migration).

Crouch, C. (2011). "Beyond the flexibility/security trade-off: reconciling confident consumers with insecure workers." British Journal of Industrial Relations (forthcoming)

Cea D'ancona, M. A. (2009). “La compleja detección del racismo y la xenofobia a través de encuesta. Un paso adelante en su medición." REIS, $\mathrm{n}^{\circ}$ 125, pp.13-45

Cea D’ancona, M.A. (2002). "La medición de las actitudes ante la inmigración: Evaluación de los indicadores tradicionales de racismo." REIS, nº 99, pp. 87-112

Cea D'ancona, M.A.; Vallés, M.S. (2010). Evolución del racismo y la xenofobia en España. Informe 2010. Ministerio de Trabajo e Inmigración, http://www.mtin.es

Ceobeanu, A.M.; Escandell, X. (2010). "Comparative Analyses of Public Attitudes Towards Immigration and Immigration Using Multinational Survey Data: A Review of Theories and Research". Annual Review of Sociology, 36: 309-28.

Citrin, J.; Green, D.P.; Muste, C.; WONG, C. (2005). "Public opinion towards immigration reform: The role of economic motivation." The Journal of Politics, vol. $59, \mathrm{n}^{\circ} 3$, pp. 858-881

EUROPEAN COMMISSION (2004). The transitional arrangements for the free movement of workers from new Members States following enlargement of the European Union on 1 of May 2004. http.//ec.europa.eu/employment_social/free movement/docs/transition

EUROPEAN COMMISSION (2007). Towards a common immigration policy. Luxemburg, COM (2007) 628 final.

EUROPEAN COMMISSION (2008). The labour market situation and impact for recent country migrants. [EU: Employment in Europe. Brussels: European Commission.] 
EUROPEAN COMMISSION (2009). Employment in Europe. Luxemburg: http//ec.europa.employment

Esping-Andersen, G. (2000). Los fundamentos sociales de las economías postindustriales. Barcelona: Ariel.

González, M. (2008) Los sindicatos ante la inmigración. Madrid: Observatorio Permanente de la Inmigración.

Habermas, J. (2000). La constelación postnacional. Barcelona: Paidós.

Hernández, D. (2012). Formación preventiva e inmigración en el sector de la construcción. Tesis Doctoral: Universidad Autónoma Barcelona.

Hainmueller, J.; Hiscox. M. J. (2007). "Educated Preferences: Explaining Attitudes Towards Immigration in Europe." International Organisations, ${ }^{\circ}$ 51, 00. 399-442.

Innerarity, C.; Acha, B. (2010). "Los discursos sobe ciudadanía e inmigración en Europea: Universalismo, extremismo y educación.” Revista Política y Sociedad, vol. 47, no 2, pp.63-84.

Jodar, P.; Alós, R.; Boso, A.; Garrell, D.; Guiu, J. (2011). L’afiliació estrangera a CCOO de Catalunya. Barcelona: Edita CERES-CCOO.

Kogan, I. (2003). "A Study of Employment Careers of Immigrants in Germany". Working Paper. University of Mannheim. Working Paper.

Mayda, A.M. (2006). "Who is Against Immigration?". Review of Economic and Statistics, August 88 (2006), pp.510-530.

Mau, S.; Burkhardt, C. (2009). "Migration and welfare state solidarity in Wetern Europe." Journal of European Social Policy, 19; 213

Menz, G. (2008). "Migration and the Welfare State". In C. Parsons and T. Smeeding (eds) Immigration and the Transformation of Europe. Cambridge: Cambridge University Press.

Morissens, A. (2008). "Immigrants, Unemployment and Europe's Varying Welfare Regimes". In C. Parsons and T. Smeeding (eds) Immigration and the Transformation of Europe. Cambridge: Cambridge University Press

Mota, F. (2005): "España: sociedad y política en perspectiva comparada". En Torcal, M.; Morales, L.; Pérez-Nieva, S. (eds).Mismo título. Valencia: Tirant lo Blanc, pp, 203-207. 
Ortega, F.; Polavieja, J.G. (2009). "Labour-market exposure: as a determinant of attitudes toward immigration." Madrid: Working Paper 2009/245. Instituto Juan March de Estudios e Investigaciones.

Pajares, M. (2008). "Foreign workers and trade unions: the challenges posed." Transfer Review, vol. 14, n. 4, pp. 607-624

Pajares, M.; Jubany, O. (2011). Sindicatos e inmigración en Europa, 1990-2010. Barcelona: Icaria y Antrazyt.

Rink, N.; Phalet, K.; Swyegedouw, M. (2009). "The effect of Immigrants Populations Size, Unemployment and Individual Characteristics on voting for the Vlaams Blok in Flanders", 1991-1999. European Sociological Review, vol. 25, $n^{\circ} 4,414-424$

Reyneri, E. (2006). "De la economía sumergida a la devaluación profesional: nivel educativo e inserción en el mercado de trabajo de los inmigrantes en Italia." REIS, $n^{\circ} 116$, pp. 215-235.

Rodríguez Monter, M. (2009). "Los valores culturales y el fenómeno de las migraciones internacionales." Athenea Digital, $\mathrm{n}^{\circ}$ 15, 217-228

Sanderson, S.K. (2004). "Ethnic Heterogeneity and Public Spending: Testing the Evolutionary Theory of Ethnicity with Cross-national Data." In Salter, F.K. (ed). Welfare, evolutionary Theory. London/Portland, OR: Frank Cass.

Saxton, G.D.; Benson, M.A. (2003). "The Origins of Socially and Politically Hostile Attitudes towards Immigrants and Outgroups: Economics, Ideology or National Context?". The Journal of Political Science, vole, 31: 101-137

Simon, R.J.; Sikich, K.W. (2007). "Public Attitudes towards Immigration and Immigration Policies across Seven Nations." International Migration Review, 41, 3, pp. $956-962$

Solé, C. ; Parella; S. Alarcón, A.; Bergalli, V.; Gibert, F. (2000). "El impacto de la inmigración en la sociedad receptora." REIS, n 90, 131-157

Soroka, S. Banting, K.; Jhonston, R. (2006). "Immigration and Redistribution in a Global Era." In Bardhan, P.; Bowles, S. and Wallerstein, M. Globalization and Egalitarian Redistribution. Princeton, NJ: Princenton University Press.

Schwartz, S.H. (1994). "Beyond individualism/collectivism: new dimensions of values”. En Kim, U.; Traindis, H.C.; Kagticibasi, C.; Choi, S.C.; Yoon, G. (ed). 
Advances in Experimental Social Psycology (vol. 25, pp. 1-65. Orlando, Fl: Academic Press.

Schwartz, S.H. (2001). "Value hierarchies across cultures. Talking a similarities perspective." Journal of Cross-Cultural Psychology, 32(3), 288-290

Schwartz, S.H. (2007). "Universalism Values and Inclusiveness of Our Moral Universe". Journal of Cross-cultural Psychology, $\mathrm{N}^{\mathrm{o}}$ 37:711. Doi $10.1177 / 0022022107308922$

Tubergen, F. (2008). "Occupational status of immigrants in cross-national perspective: A multilevel analyses of seventeen Western societies." En Parsons, C. A.; Smeeding, T. Immigration and the transformation of Europe. Cambridge: Cambridge University Press.

Zimmermann, K. (2009). "Labour mobility and integration of European labour markets." In Nowotny, E. et al: The integration of European Labour Markets. Cheltenham: Edward Elgar Publishing 